\title{
Gauge Theory and String Theory; An Introduction to the AdS/CFT Correspondence*
}

\author{
Hirosi Ooguri ${ }^{\dagger}$ \\ Caltech - USC Center for Theoretical Physics, Mail Stop 452-48 \\ California Institute of Technology, Pasadena, CA 91125, USA
}

\section{Introduction}

In this talk, I would like to show you some of the recent developments in superstring theory, in particular the relation between gauge theory and string theory. String theory was originally invented as a theory of hadrons, but it was superseded by the gauge theory. It then found its employment in quantum gravity. Now it seems that string theory and gauge theory are meeting again, and I hope this new direction will provide an interesting arena where lattice gauge theorists and string theorists can interact and exchange ideas, benefiting both.

Let me begin my talk by posing a question:

\section{What is the string theory?}

Until recently, one of the serious defects of the string theory had been that we did not know what it is. We did not have a definition of the theory. This was an unpleasant situation since there was a logical possibility that the string theory might not exist after all, even as a purely theoretical framework, let alone the possibility of describing the real world. Fortunately we now know that string theory exists in certain situations, and it is what I would like to tell you today. But before we get into that, let me tell you what we knew before.

The original "definition" of the string theory was entirely in terms of the perturbative expansion. We knew that the theory, if exists, should

\footnotetext{
*Plenary talk at LATTICE 99 held in Pisa, Itay from June 29 to July 3, 1999. To appear in the Proceedings of the conference.

${ }^{\dagger}$ On leave of absence from University of California, Berkeley.
}

contain oscillating strings, which in the limit of the vanishing coupling constant $g_{\text {string }} \rightarrow 0$, freely propagate in spacetime. We also knew how to compute string amplitudes using the perturbative expansion in $g_{\text {string }}$. In superstring, each term in the perturbative expansion was known to be finite. However this, by itself, cannot be a complete definition. The perturbative series is not convergent in most of the cases. So, even though superstring theory was proposed as the unified theory including gravity, we could not use it to study effects in strong gravitational fields to address mysteries of quantum gravity. There was a concern that there may be some unknown strong coupling phenomenon which makes the theory ill-defined.

\section{Does string theory exist?}

Recently a remarkable correspondence between gauge theory and string theory was discovered [1]3] (see [4] for a review) and it has partially resolved this problem. It also realized the earlier expectation that the 't Hooft large- $N$ limit of gauge theory is a string theory [5]. The realization, however, came with a twist - that the string theory and the gauge theory live in different dimensions. The correspondence was discovered in the study of black hole in string theory.

\section{Black $p$-branes in string theory}

It has been recognized for a long time that quantizing gravity is not a straightforward task. There are many aspects of quantum gravity which we do not know how to deal with in the standard field theory method. If string theory truly unifies quantum mechanics and general relativity, string 
theory should be able to address these puzzles of quantum gravity.

One of the important questions in quantum gravity is the information paradox of black hole. The problem is roughly as follows. The black hole is characterized by the presence of an event horizon, which separates its interior region from an outside observer. Now suppose we throw some object into the black hole. Once the object passes the horizon, we won't be able to access to the information carried by the object. In classical general relativity, the information is not lost, but is merely hidden behind the horizon. In quantum mechanics, however, the situation is different. It was showed by Hawking [6], based on the semi-classical approximation, that the black hole emits pure thermal radiation, namely with the radiation with maximum randomness. So it seems that the information carried by the object is completely dissipated as the thermal radiation. The loss of information may not be a problem in classical physics, but it is a big problem in quantum mechanics since it means that we cannot maintain the quantum coherence under the time evolution. It violates the basic axioms of quantum mechanics. This is the information paradox of quantum black hole.

String theory in fact has a large class of black hole solutions. They were called black $p$-branes, where $p$ is an integer and it can be $0,1,2$ etc. The 0-brane is the standard black hole, which is a point source. The spacetime becomes flat when one goes away from the center, but it is strongly curved near the center and there is a horizon. When $p=1$, we have the black 1-brane. It is a string-like source. It is spread in one-spatial dimensions. Combined with the time direction, the source looks like a two-dimensional surface. The 2 -brane is a membrane. In general, the $p$-brane is extended in $p$-spatial directions and 1-time direction.

So the string theory indeed have black hole solutions. To address the question of the information paradox, one needs to know what happens if we throw a string into the black $p$-brane.

There are two ways to approach this problems. One is to study the propagation of the string in the black $p$-brane geometry. There is, how- ever, another way, that is to use the collective coordinates of the $p$-brane. In this approach, we first identify intrinsic degrees of freedom of the $p$-brane, which describe the motion and the fluctuation of the brane. One can then try to formulate the problem as interactions between the string and the collective coordinates of the $p$ brane. This approach was taken by Polchinski [7], and he called this description of the $p$-brane as Dbrane. In a certain situation, in particular when we study physics at low energy, this D-brane description tells you that the dynamics of the $p$ brane is described by a gauge theory in $(p+1)$ dimensions

So we have these two descriptions of the same object. In one way of viewing at it, we have strings moving in the background geometry of black $p$-brane. In another way of viewing at it, we have the collective coordinates of the $p$-brane, which is the $(p+1)$-dimensional gauge theory, interacting with strings. The equivalence of these two descriptions is the origin of the connection between gauge theory and string theory. In the past few years, various evidences have emerged in support of the idea that gauge theory and quantum gravity are closely related. At the same time, the gauge theory description has provided us very strong computational tools to study string theory.

\section{AdS/CFT correspondence}

The evidences in support of the correspondence between gauge theory and string theory have crystallized in the work of Maldacena. He formulated the conjecture that superstring theory in a curved 10-dimensional space, which is 5dimensional anti-de Sitter space $\left(A d S_{5}\right)$ times 5dimensional sphere $\left(S^{5}\right)$, is equivalent to a gauge theory in 4 dimensions with $N=4$ supersymmetry. The anti-de Sitter space is a homogeneous space with negative curvature, which I will describe in more detail below. The gauge theory with $N=4$ supersymmetry is a conformal field theory (CFT). Thus the conjecture by Maldacena is called the AdS/CFT correspondence.

To formulate the conjecture, Maldacena looked at the black 3-brane. The D-brane description suggests that the low energy physics of the 3- 
brane is described by the gauge theory, in this case the 4-dimensional gauge theory with $N=4$ supersymmetry. This description becomes exact in the low energy limit. He then noticed that, in precisely the same limit, the black hole description also becomes nice; in this limit, the region near the event horizon of the 3-brane is amplified. The geometry near the horizon is that of $A d S_{5} \times S^{5}$. In this way, the essence of the correspondence between gauge theory and string theory has been extracted.

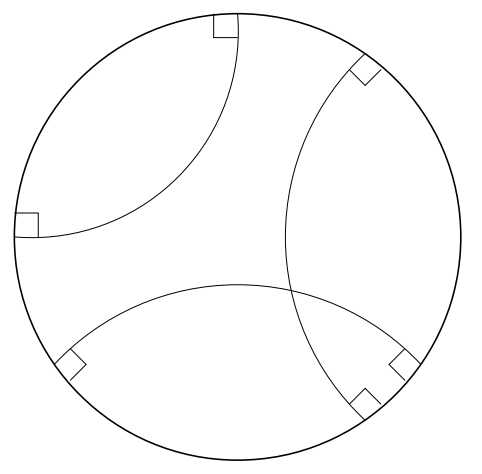

Figure 1. Poincare disc model of the hyperbolic space. Geodesics are represented by semi-circles which intersects with the boundary in the right angle.

Let me describe what the anti-de Sitter space is. The 5-dimensional anti-de Sitter space $\left(A d S_{5}\right)$ has 4 spatial dimensions and 1 time. The spacelike section of $A d S_{5}$ is simply the hyperbolic space of Lobachevsky and Bolyai. It is historically the first counter-example to Euclid's 5th postulate about parallel lines. In Figure 1, I show Poincare's disk model of the hyperbolic space. In this model, geodesics are represented by semicircles which are intersecting with the boundary in the right angle. In our case, the disk is 4 dimensional, so its boundary is 3-dimensional sphere. $A d S_{5}$ is obtained by simply adding 1 time direc-

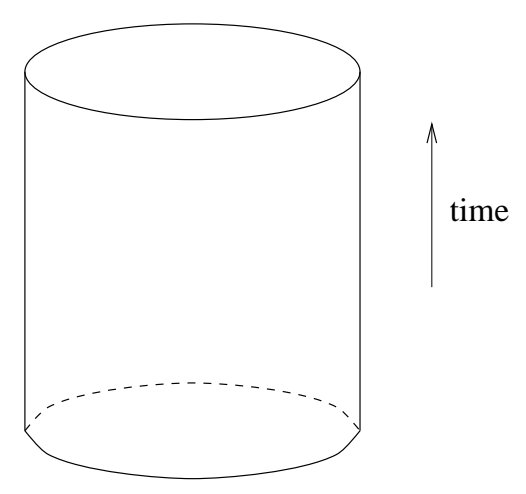

Figure 2. $A d S_{5}$ is obtained by adding one time direction to the 4-dimensional hyperbolic space.

tion to this. You may view it like a solid cylinder as in Figure 2. The boundary of $A d S_{5}$ is 4 dimensional space of $R \times S^{3}$, and it is identified as a spacetime for the gauge theory.

More precisely, the conjecture states that Type IIB superstring theory (consisting only of closed strings with the same chirality in both left and right moving sectors on the worldsheet) is equivalent to the $\mathcal{N}=4$ supersymmetric gauge theory in 4 dimensions with gauge group $S U(N)$. Type IIB superstring on $A d S_{5} \times S^{5}$ has three dimensionful parameters, $l_{A d S}, l_{\text {string }}$ and $l_{\text {Planck. }}$. By the stringy generalization of Einstein's equation, the radius of $S^{5}$ is required to be the same as the curvature radius $l_{A d S}$ of $A d S_{5}$. The string length $l_{\text {string }}$ characterizes the size of the zero point oscillation of the string, i.e. (string tension) $=$ $l_{\text {string }}^{-2}$. Finally the Planck length $l_{\text {Planck }}$ in 10 dimensions is expressed in the combination of the string coupling constant $g_{\text {string }}$ and the string length as $l_{\text {Planck }}=g_{\text {string }}^{1 / 4} l_{\text {string }}$. The theory is then characterized by two dimensionless combinations of these, for example $l_{A d S} / l_{\text {string }}$ and $L_{\text {AdS }} / l_{\text {Planck. }}$ On the other hand, the gauge theory also has two parameters, the size of the gauge group $N$ and the gauge coupling constant $g_{\text {gauge }}$. (Since the $\mathcal{N}=4$ theory is ultraviolet finite, $g_{\text {gauge }}$ is really a constant.) These two sets of 
parameters are related as

$$
\frac{l_{\text {AdS }}}{l_{\text {string }}}=\left(g_{\text {gauge }}^{2} N\right)^{1 / 4}, \quad \frac{l_{\text {AdS }}}{l_{\text {Planck }}}=N^{1 / 4} .
$$

In particular, if we take the limit $N \rightarrow \infty$ keeping $g_{\text {gauge }}^{2} N$ finite, quantum gravity effects in $A d S_{5} \times S^{5}$ are suppressed and we have strings freely propagating in the spacetime. This realizes the idea that the 't Hooft large- $N$ limit of gauge theory is a tree-level string theory.

After Maldacena's work, the conjecture was formulated in a more precise manner and various tests of this conjecture have been made. The AdS/CFT correspondence states that the two quantum theories, namely the string theory in 10 dimensions and the gauge theory in 4 dimensions are equivalent. This means that, first of all, the Hilbert space of the two theories must be identical. The Hilbert space of the string theory contains gravitons and strings propagating in the AdS space, and black holes and various black pbranes. On the other hand, the Hilbert space of the gauge theory is constructed from the gauge invariant observables such as the field strength of the gauge field. So there has to be a dictionary between the two. In fact, we have succeeded in identifying what the gravitons correspond to in the gauge theory side, and we have also understood some aspects of black holes from the gauge theory point of view. The dictionary of the two Hilbert spaces is still incomplete, and there are many things to be understood here.

The quantum theories are characterized by the operator algebras on the Hilbert spaces. Thus the equivalence of the two theories implies that the correlation functions of the two theories must be the same. After the conjecture was formulated, various computations have been done on both side, and many non-trivial agreements have been found. String theory computations also give various theoretical prediction about the gauge theory, and these are currently being examined using the gauge theory method.

There are two surprising features of the AdS/CFT correspondence. The first surprise is that string and the gauge theory live in different spacetime dimensions. I should point out that this is not the same as the Kaluza-Klein reduc- tion. In the Kaluza-Klein mechanism, one curls up a part of the spacetime into a tiny circle. If we ignore field fluctuation on the circle (or compact manifolds, in general), the dimensionality of the space is reduced. Here we are not truncating a theory. The equivalence states that the string theory is fully equivalent to the gauge theory, without any reduction. The second surprise is that string theory contains gravity and the gauge theory doesn't. These two surprises seem to be related to an earlier observation on quantum gravity by 't Hooft [8] and Susskind [9]. They suggested, based on properties of black hole, that in quantum gravity the information of the theory can be stored in lower dimensions. This idea is called Holography of Quantum Gravity. It seems that the AdS/CFT correspondence is an explicit realization of this idea.

\section{Summary and Outlook}

Let me summarize what have been accomplished. I think that the most important fact coming out from this development is that we now have a complete definition of the string theory in certain cases. For superstring on $A d S_{5} \times S^{5}$, the 4-dimensional gauge theory with $N=4$ supersymmetry can in principle give the non-perturbative definition. In addition, the AdS/CFT correspondence realizes the idea that the 't Hooft large- $N$ limit of the $S U(N)$ gauge theory is string theory. It also realizes the idea that quantum gravity is holographic.

I would like to close this talk by pointing out some future directions. Since we know string theory and gauge theory are equivalent, we may try to use string theory to do difficult computations in gauge theory or vice versa, and thereby doubling our theoretical knowledge. In fact string theory computations have shown us various new results about gauge theories with conformal symmetry. For applications to QCD, to go beyond the qualitative analysis, we need to understand string dynamics in the curved background better [10,11, and I hope there will be some progress in this direction.

One should also hope to learn much more about quantum gravity using gauge theory. Some as- 
pects of quantum black holes have been studied using the gauge theory method, in particular the microscopic derivation of the black hole entropy by Strominger and Vafa 12 .

The correspondence has so far been limited to the case when string theory is on a space which is asymptotically anti de Sitter. It is desirable to extend this to other geometry such as asymptotically flat space. More generally, one should hope to find a formulation of string theory independently of its background geometry.

The main aim of string theory research is still a search for the unified theory. In the course of this research, we have found that string theory is also useful to study various gauge theories. I hope that useful collaborations between lattice gauge theorists and string theorists would emerge from this correspondence.

\section{Acknowledgments}

I would like to thank the organizers of LATTICE 99 for the very stimulating conference and for their hospitality. This research was supported in part by NSF grant PHY-95-14797, DOE grant DE-AC03-76SF00098, and the Caltech Discovery Fund.

\section{REFERENCES}

1. J. Maldacena, Adv. Theor. Math. Phys. 2 (1998) 231-252.

2. S.S. Gubser, I.R. Klebanov, and A.M. Polyakov, Phys. Lett. B428 (1998) 105-114.

3. E. Witten, Adv. Theor. Math. Phys. 2 (1998) 253-291.

4. O. Aharony, S.S. Gubser, J. Maldacena, H. Ooguri, and Y. Oz, to appear in Phys. Rep.; hep-th/9905111.

5. G. 't Hooft, Nucl. Phys. B72 (1974) 461.

6. S.W. Hawking, Nature (1974) 30.

7. J. Polchinski, Phys. Rev. Lett. 75 (1995) 4724.

8. G. 't Hooft, Proceedings of Salamfest 1993; gr-qc/9310026.

9. L. Susskind, J. Math. Phys. 36 (1995) 6377.

10. E. Witten, Adv. Theor. Math. Phys. 2 (1998) 505.
11. D.J. Gross and H. Ooguri, Phys. Rev. D58 (1998) 106002.

12. A. Strominger and C. Vafa, Phys. Lett. B379 (1996) 99. 